\section{In properly selected patients with differentiated thyroid cancer, antithyroglobulin antibodies decline after thyroidectomy and their sole presence should not be an indication for radioiodine ablation}

Luis Felipe Zavala', María Inés Barra ${ }^{1 *}$, Roberto Olmos", Michael Tuttle², Hernán González², Nicolás Droppelmann³, Lorena Mosso', José M. Domínguez'

\begin{abstract}
Objective: Our objective was to evaluate the trend of antithyroglobulin antibodies (TgAb) during follow-up of patients with differentiated thyroid cancer (DTC) treated without RAl, as well as their role in the risk of recurrence. Subjects and methods: This was a prospective, descriptive study. A total of 152 consecutive patients with DTC treated in a single institution undergoing total thyroidectomy without RAl and followed for a median of 2.3 years (0.5-10.3) were divided in two groups: $\mathrm{TgAb}(-)$ $(n=111)$ and $\operatorname{TgAb}(+)(n=41)$. Patients were classified according to AJCC $7^{\text {th }}$ and $8^{\text {th }}$ editions, as well as to their risk of recurrence and response to treatment categories. Results: Both groups, $\operatorname{TgAb}(-)$ and $\operatorname{Tg} \mathrm{Ab}(+)$, were similar regarding patient and tumor characteristics. At the end of follow-up, 90 $(59.2 \%), 57(37.5 \%), 3(2 \%)$ and $2(1.3 \%)$ patients achieved excellent, indeterminate, biochemically incomplete and structurally incomplete response, respectively. The risk of structural recurrence was similar in both groups (TgAb[-] 0.9\% vs. TgAb[+] 2.4\%, p = 0.46). In the TgAb(+) group, TgAb became negative in $10(24.4 \%)$, decreased $\geq 50 \%$ without negativization in $25(60.9 \%)$, decreased $<50 \%$ in 4 $(9.8 \%)$ and remained stable or increased in $2(4.9 \%)$ cases. The only incomplete structural response had increasing TgAb during follow-up. Conclusions: In properly selected patients with DTC, TgAb concentration immediately after total thyroidectomy should not mandate RAI ablation, and their trend during follow-up may impact the risk of recurrence. Arch Endocrinol Metab. 2019;63(3):293-9
\end{abstract}

Keywords

Thyroid cancer; antithyroglobulin antibodies; radioiodine ablation
1 Departments of Endocrinology, Faculty of Medicine, Pontificia Universidad Católica de Chile, Santiago, Chile ${ }^{2}$ Department of Endocrinology Service, Memorial Sloan-Kettering Cancer Center, New York, New York ${ }^{3}$ Head and Neck Surgery, Faculty of Medicine, Pontificia Universidad Católica de Chile, Santiago, Chile

* These two co-authors contributed similar between them in the development of this article

Correspondence to: José Miguel Domínguez Diagonal Paraguay, 362 , 4th floor, Santiago, Chile jdomingu@uc.cl

Received on Nov/25/2018 Accepted on Feb/17/2019

DOI: 10.20945/2359-3997000000123

\section{INTRODUCTION}

$\mathrm{D}$ ifferentiated thyroid cancer (DTC) is the most common endocrine malignancy, and its incidence has increased in the last decades due, in part, to expanded availability of neck ultrasonography (US) (1-3). Because most of these newly diagnosed tumors have low risk of recurrence, there is a need to personalize their management to minimize the adverse effects of therapy (4).

The treatment of DTC includes surgery, selective use of radioactive iodine (RAI) and levothyroxine supplementation (4). RAI has proven to be effective in patients with intermediate and high risk of recurrence, while its utility has been questioned in low-risk patients (5). Because one of the benefits of RAI is to facilitate patient follow-up, its use has been suggested in the presence of elevated antithyroglobulin antibodies $(\mathrm{TgAb})$ to enable correct interpretation of serum thyroglobulin (Tg) (6). However, RAI use is associated with short- and long-term adverse effects, which can significantly impair patients' quality of life. For this reason, the indication of RAI must be adjusted to the patient's individual risk of recurrence and mortality (7-9).

After initial treatment, follow-up of patients with DTC includes measurement of serum Tg and imaging studies according to the initial risk of recurrence (10-12). About $20 \%$ of patients have $\operatorname{TgAb}(+)$, 
which can interfere with $\mathrm{Tg}$ measurement, usually underestimating their concentration and thereby potentially leading to false-negative results (13-17). For this reason, most studies evaluating treatment and follow-up of patients with DTC (with or without $\mathrm{RAI}$ ) exclude $\operatorname{TgAb}(+)$ cases (18). The $\mathrm{TgAb}$ are not direct tumor markers, and their concentration reflects the immune response to changes in the $\mathrm{Tg}$ antigen, secondary to benign or malignant disease (19). To estimate real changes in their concentration, $\operatorname{TgAb}$ usually require a minimum follow-up of 6 months, during which the trend in their concentration appears to be more accurate as a marker of disease rather than the absolute level itself (19). For instance, in patients with DTC and $\operatorname{TgAb}(+)$ treated with RAI, a reduction greater than $50 \%$ in $\mathrm{TgAb}$ concentration during the first 12 months of follow-up has been associated with a better prognosis $(20,21)$. Matrone and cols. recently published a prospective follow-up of $\operatorname{TgAb}(+)$ patients with microcarcinomas treated without RAI (22). To the best of our knowledge, no other prospective study has compared the clinical outcome of patients with DTC treated without RAI based on the presence or absence of $\mathrm{TgAb}$.

The aim of this study was to prospectively evaluate the trend of $\mathrm{TgAb}$ concentration during follow-up of patients with DTC treated without RAI, as well as their impact on the risk of recurrence and response to treatment.

\section{SUBJECTS AND METHODS}

\section{Subjects}

This study was approved by the Ethics Committee of Pontificia Universidad Católica de Chile.

Patients 18 years old or older, consecutively treated and followed prospectively at our institution between December 2012 and December 2017, who met the following criteria were initially selected: (i) diagnosis of DTC; (ii) subjected to total thyroidectomy, with neck dissection performed only with evidence of clinically apparent nodal disease detected at clinical examination, preoperative neck US or during intraoperative inspection by the surgeon; and (iii) followed for at least 6 months with at least 2 measurements of $\mathrm{Tg}$ and $\mathrm{TgAb}$. Nodal disease was classified in low and high volume according to 2015 ATA guidelines (4).
The need for RAI was defined in each patient following the recommendations of the 2015 ATA guidelines (4). Patients were classified according to ATA recurrence risk category (low, intermediate and high) as well as the $7^{\text {th }}$ and $8^{\text {th }}$ editions of the AJCC/ UICC staging system (I, II, III and IV) based on the preoperative neck US, intraoperative findings and final surgical pathology report (23).

Patients that met any of the following criteria were excluded: (i) partial thyroidectomy; (ii) absence of or incomplete surgical pathology report; (iii) follow-up of less than 6 moths; and (iv) Tg, TgAb or US not performed during follow-up. From the 180 initially included patients, 28 were excluded due to of lack of follow-up $(\mathrm{n}=13)$ or insufficient clinical and pathological information $(\mathrm{n}=15)$.

\section{Follow-up}

After initial surgery, all patients were treated with a levothyroxine (LT4) dose sufficient to maintain a serum thyrotropin $(\mathrm{TSH})$ concentration $<1.0 \mathrm{UI} / \mathrm{mL}$. The patients underwent clinical examination, $\mathrm{Tg}, \mathrm{TgAb}$ and neck US every 6 months during the first year and thereafter at 6-to 12 -month intervals at their attending physician's discretion.

\section{Outcomes}

Cervical neck US was performed by experienced radiologists. Negative neck US was defined as either normal exploration or the presence of any of the following findings: (i) thyroid bed avascular nodules $\leq 5$ $\mathrm{mm}$ in diameter, stable during follow-up or (ii) reactive cervical lymph nodes. Disease recurrence/persistence was defined as suspicious findings in images, confirmed with cytopathologic study and Tg measurement on aspirate.

Response to treatment was defined according to US, $\mathrm{Tg}$ and $\mathrm{TgAb}$ findings during follow-up. Excellent response was defined when all of the following were present: negative US, $\mathrm{Tg}<0.2 \mathrm{ng} / \mathrm{mL}$ and negative $\mathrm{TgAb}$. Incomplete biochemical response was defined in the presence of negative US and either non-stimulated $\mathrm{Tg}>5 \mathrm{ng} / \mathrm{mL}$ plus negative $\mathrm{TgAb}$, or positive $\mathrm{TgAb}$ with an increase in concentration. Incomplete structural response was defined as highly suspicious neck US (or any other image modality), confirmed through cytological study, regardless of $\mathrm{Tg}$ or $\mathrm{TgAb}$ serum concentration. Indeterminate response was defined either as (i) negative neck US plus $\mathrm{Tg} \geq 0.2$ - 
$\leq 5 \mathrm{ng} / \mathrm{mL}$ plus $\operatorname{TgAb}(-)$ or (ii) negative US plus $\operatorname{TgAb}(+)$ with decreasing or stable concentration during follow-up or (iii) nonspecific findings through imaging studies (24). To refine the evaluation of the impact of $\mathrm{TgAb}$ on prognosis, we classified the $\mathrm{TgAb}$ concentration decrease during follow-up as $<50 \%$ or $\geq 50 \%$.

\section{Assays}

$\mathrm{Tg}$ was measured using a chemiluminescent immunoassay with functional sensitivity of $0.1 \mathrm{ng} / \mathrm{mL}$ (Elecsys II, Roche Diagnostics, Rotkreutz, Switzerland). $\mathrm{TgAb}$ were also measured using a chemiluminescent immunoassay (Architect il000, Abbot Laboratories, Abbott Park, IL), with a reference value of up to $4.11 \mathrm{IU} / \mathrm{mL}$ and analytical sensitivity of 1.0 $\mathrm{IU} / \mathrm{mL}$. For the purpose of this study, every value below 4.1 UI/mL was considered negative. Both $\mathrm{Tg}$ and $\mathrm{TgAb}$ were normalized with CRM 457.

\section{Statistical analysis}

Continuous variables are presented either as mean and standard deviation or median with range, as appropriate. Categorical comparisons were performed using Fisher's exact test, and continuous variables were compared using Student's t-test. A p-value of $<0.05$ was considered significant. Statistical analysis was performed using SPSS (v.15.0.0: SPSS, Inc., Chicago, IL).

\section{RESULTS}

A total of 152 patients with a mean age of 40.5 (18-80) years, followed for a median of $2.3(0.5-10.3)$ years were included. Total thyroidectomy alone was performed in $124(81.6 \%)$ patients, and total thyroidectomy with lymph node dissection in 28 (18.4\%). Papillary thyroid cancer was the histological diagnosis in $150(98.7 \%)$ and follicular thyroid cancer in $2(1.3 \%)$, both cases minimally invasive and less than $4 \mathrm{~cm}$. Of the total cohort, 97 (64\%) had microcarcinoma, $54(35.5 \%)$ were multicentric, 40 $(26.3 \%)$ were bilateral and $13(8.5 \%)$ had lymph node metastases: 8 with low-volume and 5 with high-volume disease, with highest nodal diameter of $0.5 \mathrm{~cm}$ and no cases of extra-nodal extension (Table 1).

According to the ATA 2015 guidelines, 123 $(80.9 \%)$ and 29 (19.1\%) patients were classified as low and intermediate risk of recurrence, respectively. No patients were classified as high risk of recurrence. According to the AJCC $7^{\text {th }}$ edition, 138 (90.8\%) patients were classified as stage I, and according to the AJCC $8^{\text {th }}$ edition, $100 \%$ of the cohort was classified as stage I (Table 1).

A median of 4.4 (2.0-7.0) $\mathrm{Tg}$ and $\mathrm{TgAb}$ measurements were performed. Forty-one (27\%) patients had $\operatorname{TgAb}(+)$ at diagnosis. Their clinical characteristics were similar to patients with $\operatorname{TgAb}(-)$ including age, proportion of microcarcinomas and cases classified either as stage I according to the AJCC $7^{\text {th }} 8^{\text {th }}$ editions or low risk of recurrence according to the ATA 2015 guidelines (Table 1).

During the study, $2(1.3 \%)$ patients of the whole cohort developed structural incomplete response, while $102(67.1 \%)$ patients achieved excellent response at some point during follow-up. Of this last subgroup, no patients developed structural disease, while at the end of follow-up, $90(88 \%)$ patients

Table 1. Characteristics of the 152 patients studied

\begin{tabular}{|c|c|c|c|c|}
\hline & \multirow{2}{*}{ Total cohort $(\mathrm{N}=152)$} & \multicolumn{2}{|c|}{ Antithyroglobulin Antibodies } & \multirow{2}{*}{ p-value } \\
\hline & & Negative $(n=111)$ & Positive $(n=41)$ & \\
\hline Age (years) & $40.5(18-80)$ & $42(18-80)$ & $35(18-63)$ & 0.25 \\
\hline Follow-up (years) & $2.3(0.5-10.3)$ & $2.3(0.5-10.3)$ & $2.3(0.5-9.8)$ & 0.87 \\
\hline Microcarcinoma & $97(64 \%)$ & $72(75 \%)$ & $25(61 \%)$ & 0.70 \\
\hline \multicolumn{5}{|l|}{ AJCC VII edition } \\
\hline I & $138(90.8 \%)$ & 99 (89.2\%) & 39 (95.2\%) & \multirow{3}{*}{0.375} \\
\hline$\|$ & $3(2 \%)$ & $2(1.8 \%)$ & $1(2.4 \%)$ & \\
\hline III & $11(7.2 \%)$ & $10(9 \%)$ & $1(2.4 \%)$ & \\
\hline \multicolumn{4}{|l|}{ AJCC VIII edition } & \multirow{2}{*}{1} \\
\hline । & $152(100 \%)$ & $111(100 \%)$ & $41(100 \%)$ & \\
\hline \multicolumn{5}{|l|}{ ATA (2015) } \\
\hline Low risk & $123(80.9 \%)$ & $90(81.1 \%)$ & $33(80.5 \%)$ & \multirow{2}{*}{0.93} \\
\hline Intermediate risk & $29(19.1 \%)$ & $21(18.9 \%)$ & $8(19.5 \%)$ & \\
\hline
\end{tabular}


sustained excellent response and 12 patients were classified as having indeterminate response (Table 2). Of the 95 patients with initial indeterminate response, $53(55.8 \%)$ developed excellent response during follow-up at some point, while at the end of the study, $46(48.4 \%)$ achieved excellent response, $46(48.4 \%)$ indeterminate response, $2(2.1 \%)$ biochemical incomplete response and 1 (1.1\%) structural incomplete response (Table 2 ).

The rate of incomplete structural response was similar between $\operatorname{TgAb}(-)$ and $\operatorname{TgAb}(+)$ patients: $0.9 \%$ vs $2.4 \%(\mathrm{p}=0.46)$ (Table 2$)$. Regarding the 41 patients with $\operatorname{TgAb}(+), \operatorname{TgAb}$ concentration became negative in $10(24.4 \%)$, decreased $\geq 50 \%$ without negativization in $25(60.9 \%)$, decreased $<50 \%$ in $4(9.8 \%)$ and remained stable or increased in $2(4.9 \%)$ cases (Table 3$)$. Among the patients in whom $\mathrm{TgAb}$ concentration decreased, the median time to reach its nadir was 1.9 years (range 0.2 3.1; interquartile range 1.5-3.0). At the end of followup, $10(24.4 \%)$ patients achieved excellent, 28 (68.3\%) indeterminate, $2(4.9 \%)$ biochemical incomplete and 1 $(2.4 \%)$ structural incomplete response. In this group, the only incomplete structural response had increasing $\mathrm{TgAb}$ during follow-up.

The three patients with incomplete biochemical response underwent active surveillance, and none developed structural disease at the end of follow-up. In the two patients with a structural incomplete response, both had US/cytologically proven cervical nodal disease at 6 and 18 months after initial therapy. These patients underwent neck dissection and RAI and were both free of disease at the end of follow-up.

\section{DISCUSSION}

This study prospectively evaluated the clinical and biochemical evolution of patients with DTC treated with total thyroidectomy without RAI, showing that most of them $(59.2 \%)$ achieved excellent response to treatment at the end of follow-up, and only $1.3 \%$ developed structural incomplete response that required further therapy. Our cohort included patients with DTC similar to most previous studies that included patients treated without RAI: predominance of papillary thyroid cancer of initial low risk of recurrence, as well as a similar rate of biochemical and structural recurrence during follow-up $(25,26)$.

As in other studies, $27 \%$ of our patients had $\operatorname{TgAb}(+)$ at diagnosis, with a similar clinical presentation and evolution to those with $\operatorname{TgAb}(-)$, including a comparable risk of incomplete structural response during follow-up. Our findings are consistent with other reports that evaluated the impact of $\mathrm{TgAb}$ on DTC, with their presence not being associated with a

Table 2. Clinical outcomes in the whole cohort and according to the presence of anti-thyroglobulin antibodies

\begin{tabular}{|c|c|c|c|c|}
\hline & \multirow{2}{*}{ Total cohort $(n=152)$} & \multicolumn{2}{|c|}{ Anti-thyroglobulin antibodies } & \multirow{2}{*}{ p-value } \\
\hline & & Negative $(n=111)$ & Positive ( $n=41)$ & \\
\hline \multicolumn{5}{|c|}{ Structural incomplete response } \\
\hline Yes & $2(1.3 \%)$ & $1(0.9 \%)$ & $1(2.4 \%)$ & 0.46 \\
\hline No & 150 (98.7\%) & $110(99.1 \%)$ & $40(97.6 \%)$ & \\
\hline \multicolumn{5}{|l|}{$\begin{array}{l}\text { Response to treatment at the } \\
\text { end of follow-up }\end{array}$} \\
\hline Excellent & $90(59.2 \%)$ & $80(72.1 \%)$ & $10(24.4 \%)$ & \\
\hline Indeterminate & $57(37.5 \%)$ & $29(26.1 \%)$ & $28(68.3 \%)$ & $<0.01$ \\
\hline Biochemical incomplete & $3(2 \%)$ & $1(0.9 \%)$ & $2(4.9 \%)$ & \\
\hline Structural Incomplete & $2(1.3 \%)$ & $1(0.9 \%)$ & $1(2.4 \%)$ & \\
\hline
\end{tabular}

Table 3. Clinical outcome of patients with positive antithyroglobulin antibodies

\begin{tabular}{lcc}
\hline Trend of TgAb concentration during follow-up & N (\%) & Structural disease \\
\hline Negativization & $10(24.4 \%)$ & $0(0 \%)$ \\
$\geq 50 \%$ decrease without negativization & $25(60.9 \%)$ & $0(0 \%)$ \\
Stable or increase & $4(9.8 \%)$ & $0(0 \%)$ \\
\hline
\end{tabular}

TgAb: antithyroglobulin antibodies. 
higher risk of recurrence or mortality (27), as well as some studies suggesting that their concentration is not related to tumor mass $(19,27)$.

It has been previously reported that in patients with DTC and $\operatorname{TgAb}(+)$, the trend of the $\operatorname{TgAb}$ concentration during follow-up can be used as a surrogate marker of $\mathrm{Tg}$ when evaluating the response to treatment (28). Unfortunately, this information is derived from retrospective studies including patients initially treated with RAI (20). Our study exclusively included patients treated without RAI followed prospectively. In the $\operatorname{AcTg}(+)$ group, the trend in antibodies concentration during follow-up was favorable in $85.3 \%$ of cases, with negativization in $24.4 \%$ and a decrease of $\geq 50 \%$ without negativization in another $60.9 \%$. In this subgroup, there were no cases of structural recurrence, and $24.4 \%$ of patients achieved excellent response at the end of follow-up (negative neck US plus $\mathrm{TgAb}[-]$ and $\mathrm{Tg}$ $<0.2 \mathrm{ng} / \mathrm{mL}$ ). These findings are consistent with studies that included patients treated with RAI, in which a decrease $\geq 50 \%$ in $\mathrm{TgAb}$ concentration was associated with a risk of recurrence of 0 and $3.3 \%$ in lowand intermediate-risk patients, respectively $(20,29)$. Also similar to our cohort, previous studies focusing on patients with $\operatorname{TgAb}(+)$ reported a $\geq 50 \%$ decrease of their concentration in $75 \%$, with negativization in $30 \%$ of cases $(28,30)$. The similar behavior of $\mathrm{TgAb}$ in patients treated with or without RAI casts doubt on the role of RAI to facilitate follow-up after initial treatment.

Interestingly, at the end of follow-up, $75.6 \%$ of the whole cohort achieved either one of the following: i) excellent response to therapy (including initially $\operatorname{TgAb}[-]$ and $\operatorname{TgAb}[+]$ with negativization during follow-up) or ii) initially $\operatorname{TgAb}(+)$, with $\geq 50 \%$ decrease of $\operatorname{TgAb}$ without negativization, in addition to $\mathrm{Tg}$ $<0.2 \mathrm{ng} / \mathrm{mL}$ and negative US. It is well known that both aforementioned conditions are associated with a very low risk of recurrence (20). As mentioned before, $27 \%$ of patients with initial $\operatorname{TgAb}(+)$ achieved excellent response at the end of follow-up. However, a significant decrease in $\operatorname{TgAb}$ concentration, even without negativization, is also associated with an excellent prognosis. Our data, hopefully confirmed by further studies with longer follow-up, may challenge the current definition of excellent response, allowing for the inclusion of patients with $\operatorname{TgAb}(+)$, in which antibodies concentration decrease $\geq 50 \%$ (in conjunction with a negative US) during follow-up. These findings confirm that, in properly selected patients with low or intermediate risk of recurrence, in whom the use of RAI is selective, the behavior of $\mathrm{Tg}$ and $\mathrm{TgAb}$ during follow-up can modulate the indication for RAI. Furthermore, previous studies have shown that in low- and intermediate-risk patients, the efficacy of RAI is preserved even if administered up to one year after surgery, giving the clinician enough time to thoroughly decide its indication (31-33).

It is also notable that of the 95 patients with an indeterminate response at their first follow-up evaluation (representing 63\% of the entire cohort), only 1 of them developed structural disease, which was successfully treated with surgery and RAI. This finding, consistent with previous reports, suggests that in patients with DTC of low and selected intermediate risk, a conservative approach with active surveillance is safe and avoids exposure of an important number of patients to therapies with potential risks, such as RAI and surgery (25).

As previously described, most patients in our study achieved either excellent or indeterminate response. However, and despite the limited number of cases, half of the patients with stable or increasing $\operatorname{TgAb}(+)$ concentration during follow-up developed structural recurrence. Our finding, similar to previous reports, highlights the importance of closer surveillance in this specific group of patients (25).

One limitation of this study was the limited length of follow-up (median 2.3 years), which could result in underestimation of the risk of disease recurrence. However, $75.6 \%$ of our cohort achieved either excellent response or negative US plus a significant decrease in $\mathrm{TgAb}$ levels, findings that in a previous study from Momesso were associated with a risk of recurrence of only $1.3 \%$ after a median follow-up of 8.3 years $(25)$. A second limitation was the high proportion (64\%) of microcarcinomas in our cohort, considering that according to the 2015 ATA guidelines, these tumors could be submitted to active surveillance with neck US, in the absence of sonographic or cytologic signs of aggressiveness. However, in our study, we began recruitment in 2013, years before the publication of the aforementioned guidelines, when the indication of fine-needle aspiration was mainly driven by clinical and sonographic risk factors, regardless of nodule size (34).

Finally, there is still doubt concerning the definition of $\operatorname{TgAb}(+)$, with values above both the analytical sensitivity as well as the upper reference limit of the assay being considered $\operatorname{TgAb}(+)$. Regarding this 
point, a recent study did not find differences in clinical outcomes when comparing patients with $\operatorname{TgAb}<1.0$ $\mathrm{IU} / \mathrm{mL}$ and $\mathrm{TgAb}<4.11 \mathrm{IU} / \mathrm{mL}$ using the same assay as in our present study (35).

We conclude that in properly selected patients with DTC, the concentration of TgAb usually declines during follow-up after total thyroidectomy, and their sole presence should not be an indication of RAI ablation.

Funding: the authors received no specific funding for this work.

Disclosure: no potential conflict of interest relevant to this article was reported.

\section{REFERENCES}

1. La Vecchia C, Malvezzi M, Bosetti C, Garavello W, Bertuccio P, Levi $F$, et al. Thyroid cancer mortality and incidence: a global overview. Int J Cancer. 2015;136(9):2187-95.

2. Davies L, Morris LG, Haymart M, Chen AY, Goldenberg D, Morris J, et al.; AACE Endocrine Surgery Scientific Committee. American Association of Clinical Endocrinologists and American College of Endocrinology Disease State Clinical Review: the increasing incidence of thyroid cancer. Endocr Pract. 2015;21(6):686-96.

3. Lim H, Devesa SS, Sosa JA, Check D, Kitahara CM. Trends in Thyroid Cancer Incidence and Mortality in the United States, 1974-2013. JAMA. 2017;317(13):1338-48.

4. Haugen BR, Alexander EK, Bible KC, Doherty GM, Mandel SJ, Nikiforov YE, et al. 2015 American Thyroid Association Management Guidelines for Adult Patients with Thyroid Nodules and Differentiated Thyroid Cancer: The American Thyroid Association Guidelines Task Force on Thyroid Nodules and Differentiated Thyroid Cancer. Thyroid. 2016;26(1):1-133.

5. Carhill AA, Litofsky DR, Ross DS, Jonklaas J, Cooper DS, Brierley JD, et al. Long-Term Outcomes Following Therapy in Differentiated Thyroid Carcinoma: NTCTCS Registry Analysis 1987-2012. J Clin Endocrinol Metab. 2015;100(9):3270-9.

6. Chiovato L, Latrofa F, Braverman LE, Pacini F, Capezzone $M$, Masserini $L$, et al. Disappearance of humoral thyroid autoimmunity after complete removal of thyroid antigens. Ann Intern Med. 2003;139(5 Pt 1):346-51.

7. Iyer NG, Morris LG, Tuttle RM, Shaha AR, Ganly I. Rising incidence of second cancers in patients with low-risk (T1N0) thyroid cancer who receive radioactive iodine therapy. Cancer. 2011;117(19): 4439-46.

8. Mendoza A, Shaffer B, Karakla D, Mason ME, Elkins D, Goffman TE. Quality of life with well-differentiated thyroid cancer: treatment toxicities and their reduction. Thyroid. 2004;14(2): $133-40$.

9. Florenzano P, Guarda FJ, Jaimovich R, Droppelmann N, González H, Domínguez JM. Radioactive lodine Administration Is Associated with Persistent Related Symptoms in Patients with Differentiated Thyroid Cancer Int J Endocrinol. 2016;2016:2586512.

10. Tuttle RM, Tala H, Shah J, Leboeuf R, Ghossein R, Gonen M, et al. Estimating risk of recurrence in differentiated thyroid cancer after total thyroidectomy and radioactive iodine remnant ablation: using response to therapy variables to modify the initial risk estimates predicted by the new American Thyroid Association staging system. Thyroid. 2010;20(12):1341-9.
11. Castagna MG, Maino F, Cipri C, Belardini V, Theodoropoulou A, Cevenini G, et al. Delayed risk stratification, to include the response to initial treatment (surgery and radioiodine ablation), has better outcome predictivity in differentiated thyroid cancer patients. Eur J Endocrinol. 2011;165(3):441-6.

12. Pitoia F, Bueno F, Urciuoli C, Abelleira E, Cross G, Tuttle RM. Outcomes of patients with differentiated thyroid cancer riskstratified according to the American thyroid association and Latin American thyroid society risk of recurrence classification systems. Thyroid. 2013;23(11):1401-7.

13. Kumar A, Shah DH, Shrihari U, Dandekar SR, Vijayan U, Sharma SM. Significance of antithyroglobulin autoantibodies in differentiated thyroid carcinoma. Thyroid. 1994;4(2):199-202.

14. Souza SL, Montalli Da Assumpção LV, Ward LS. Ward, Impact of previous thyroid autoimmune diseases on prognosis of patients with well-differentiated thyroid cancer. Thyroid. 2003;13(5):491-5.

15. Spencer C, Petrovic I, Fatemi S, LoPresti J. Serum thyroglobulin ( $\mathrm{Tg}$ ) monitoring of patients with differentiated thyroid cancer using sensitive (second-generation) immunometric assays can be disrupted by false-negative and false-positive serum thyroglobulin autoantibody misclassifications. J Clin Endocrinol Metab. 2014;99(12):4589-99.

16. Spencer C, Petrovic I, Fatemi S. Current thyroglobulin autoantibody ( $\mathrm{TgAb}$ ) assays often fail to detect interfering $\mathrm{TgAb}$ that can result in the reporting of falsely low/undetectable serum Tg IMA values for patients with differentiated thyroid cancer. J Clin Endocrinol Metab. 2011;96(5):1283-91.

17. Spencer CA. Clinical review: Clinical utility of thyroglobulin antibody (TgAb) measurements for patients with differentiated thyroid cancers (DTC). J Clin Endocrinol Metab. 2011;96(12): 3615-27.

18. Lamartina L, Durante C, Filetti S, Cooper DS. Low-risk differentiated thyroid cancer and radioiodine remnant ablation: a systematic review of the literature. J Clin Endocrinol Metab. 2015;100(5):1748-61.

19. Verburg FA, Luster M, Cupini C, Chiovato L, Duntas L, Elisei R, et al. Implications of thyroglobulin antibody positivity in patients with differentiated thyroid cancer: a clinical position statement. Thyroid. 2013;23(10):1211-25.

20. Rosario PW, Carvalho M, Mourão GF, Calsolari MR. Comparison of Antithyroglobulin Antibody Concentrations Before and After Ablation with 131 l as a Predictor of Structural Disease in Differentiated Thyroid Carcinoma Patients with Undetectable Basal Thyroglobulin and Negative Neck Ultrasonography. Thyroid. 2016;26(4):525-31.

21. Kim WG, Yoon JH, Kim WB, Kim TY, Kim EY, Kim JM, et al. Change of serum antithyroglobulin antibody levels is useful for prediction of clinical recurrence in thyroglobulin-negative patients with differentiated thyroid carcinoma. J Clin Endocrinol Metab. 2008;93(12):4683-9.

22. Matrone A, Latrofa F, Torregrossa L, Piaggi P, Gambale C, Faranda A, et al. Changing Trend of Thyroglobulin Antibodies in Patients With Differentiated Thyroid Cancer Treated With Total Thyroidectomy Without 131। Ablation. Thyroid. 2018;28(7):871-9.

23. Tuttle RM, Haugen B, Perrier ND. Updated American Joint Committee on Cancer/Tumor-Node-Metastasis Staging System for Differentiated and Anaplastic Thyroid Cancer (Eighth Edition): What Changed and Why? Thyroid. 2017;27(6):751-6.

24. Momesso DP, Tuttle RM, Update on differentiated thyroid cancer staging. Endocrinol Metab Clin North Am. 2014;43(2):401-21.

25. Momesso DP, Vaisman F, Yang SP, Bulzico DA, Corbo R, Vaisman $M$, et al. Dynamic Risk Stratification in Patients with Differentiated Thyroid Cancer Treated Without Radioactive lodine. J Clin Endocrinol Metab. 2016;101(7):2692-700. 
26. Spencer CA, Takeuchi M, Kazarosyan M, Wang CC, Guttler RB, Singer PA, et al. Serum thyroglobulin autoantibodies: prevalence, influence on serum thyroglobulin measurement, and prognostic significance in patients with differentiated thyroid carcinoma. $J$ Clin Endocrinol Metab. 1998;83(4):1121-7.

27. McLeod DS, Cooper DS, Ladenson PW, Ain KB, Brierley JD, Fein HG, et al.; The National Thyroid Cancer Treatment Cooperative Study Group. Prognosis of differentiated thyroid cancer in relation to serum thyrotropin and thyroglobulin antibody status at time of diagnosis. Thyroid. 2014;24(1):35-42.

28. Spencer $\mathrm{C}$, Fatemi S. Thyroglobulin antibody ( $\mathrm{TgAb})$ methods Strengths, pitfalls and clinical utility for monitoring TgAb-positive patients with differentiated thyroid cancer. Best Pract Res Clin Endocrinol Metab. 2013;27(5):701-12.

29. Durante C, Tognini S, Montesano T, Orlandi F, Torlontano M, Puxeddu E, et al.; PTC Study Group. Clinical aggressiveness and long-term outcome in patients with papillary thyroid cancer and circulating anti-thyroglobulin autoantibodies. Thyroid. 2014;24(7):1139-45.

30. Görges R, Maniecki M, Jentzen W, Sheu SN, Mann K, Bockisch A, et al. Development and clinical impact of thyroglobulin antibodies in patients with differentiated thyroid carcinoma during the first 3 years after thyroidectomy. Eur J Endocrinol. 2005;153(1):49-55.
31. Suman $\mathrm{P}$, Wang $\mathrm{CH}$, Moo-Young TA, Prinz RA, Winchester DJ. Timing of Adjuvant Radioactive lodine Therapy Does Not Affect Overall Survival in Low- and Intermediate-Risk Papillary Thyroid Carcinoma. Am Surg. 2016;82(9):807-14.

32. Suman $\mathrm{P}$, Wang $\mathrm{CH}$, Abadin SS, Block R, Raghavan V, Moo-Young $\mathrm{TA}$, et al. Timing of radioactive iodine therapy does not impact overall survival in high-risk papillary thyroid carcinoma. Endocr Pract. 2016;22(7):822-31.

33. Scheffel RS, Zanella AB, Dora JM, Maia AL. Timing of Radioactive lodine Administration Does Not Influence Outcomes in Patients with Differentiated Thyroid Carcinoma. Thyroid. 2016;26(11):1623-9.

34. American Thyroid Association (ATA) Guidelines Taskforce on Thyroid Nodules and Differentiated Thyroid Cancer, Cooper DS, Doherty GM, Haugen BR, Kloos RT, Lee SL, Mandel SJ, et al. Revised American Thyroid Association management guidelines for patients with thyroid nodules and differentiated thyroid cancer. Thyroid. 2009;19(11):1167-214.

35. Côrtes MCS, Rosario PW, Oliveira LFF, Calsolari MR. Clinical Impact of Detectable Antithyroglobulin Antibodies Below the Reference Limit (Borderline) in Patients with Papillary Thyroid Carcinoma with Undetectable Serum Thyroglobulin and Normal Neck Ultrasonography After Ablation: A Prospective Study. Thyroid. 2018;28(2):229-35. 